\title{
Initiation of a lightning search using the lightning and airglow camera onboard the Venus orbiter Akatsuki
}

\author{
Yukihiro Takahashi ${ }^{*}$, Mitsuteru Sato ${ }^{1}$, Masataka Imai ${ }^{1}$, Ralph Lorenz ${ }^{2}$, Yoav Yair $^{3}$, Karen Aplin ${ }^{4}$, Georg Fischer $^{5}$, \\ Masato Nakamura ${ }^{6}$, Nobuaki Ishii ${ }^{6}$, Takumi Abe ${ }^{6,7}$, Takehiko Satoh $^{6,7}$, Takeshi Imamura $^{8}$, Chikako Hirose ${ }^{6}$, \\ Makoto Suzuki ${ }^{6}$, George L. Hashimoto ${ }^{9}$, Naru Hirata ${ }^{10}$, Atsushi Yamazaki ${ }^{6,11}$, Takao M. Sato ${ }^{6}$, Manabu Yamada ${ }^{12}$, \\ Shin-ya Murakami ${ }^{6}$, Yukio Yamamoto ${ }^{6}$, Tetsuya Fukuhara ${ }^{13}$, Kazunori Ogohara ${ }^{14}$, Hiroki Ando ${ }^{15}$, \\ Ko-ichiro Sugiyama ${ }^{16}$, Hiroki Kashimura ${ }^{17}$ and Shoko Ohtsuki ${ }^{18}$
}

\begin{abstract}
The existence of lightning discharges in the Venus atmosphere has been controversial for more than 30 years, with many positive and negative reports published. The lightning and airglow camera (LAC) onboard the Venus orbiter, Akatsuki, was designed to observe the light curve of possible flashes at a sufficiently high sampling rate to discriminate lightning from other sources and can thereby perform a more definitive search for optical emissions. Akatsuki arrived at Venus during December 2016, 5 years following its launch. The initial operations of LAC through November 2016 have included a progressive increase in the high voltage applied to the avalanche photodiode detector. LAC began lightning survey observations in December 2016. It was confirmed that the operational high voltage was achieved and that the triggering system functions correctly. LAC lightning search observations are planned to continue for several years.
\end{abstract}

Keywords: Venus, Lightning, Flash, Akatsuki, Lightning and airglow camera

\section{Introduction}

Observations detecting potential lightning flashes on Venus have been attempted for more than 30 years, with both optical and radio wave sensors, and by spacecraft and ground-based telescopes, as reviewed by Takahashi et al. (2008). However, the scientific community has not yet reached a consensus on the existence of lightning on the planet. Among the principal reasons for the ongoing controversy may be the challenges in discriminating natural lightning signatures from transient noise caused by other sources. Receivers onboard spacecraft in the very low frequency (VLF) and high frequency (HF) ranges have recorded transient signals possibly emitted from lightning channels (e.g., Russell 1991; Gurnett et al. 1991,

\footnotetext{
*Correspondence: yukihiro@sci.hokudai.ac.jp

${ }^{1}$ Graduate School of Science, Hokkaido University, Kita 10 Nishi 8, Kita-ku, Sapporo, Hokkaido 060-0810, Japan

Full list of author information is available at the end of the article
}

2001). However, such waves could also have been generated by other sources, such as from spacecraft equipment, discharge from the spacecraft body to the space environment, or space plasma instabilities. Borucki et al. (1981, 1991) investigated data acquired by the Star Tracker on the Pioneer Venus Orbiter, which was designed to detect star light to determine the attitude of the spacecraft but was also expected to be sensitive to optical lightning flashes. However, this sensor also records pulses caused by cosmic rays incident on the detector, and given the sensor's electronics cannot be distinguished from a lightning flash. The authors attempted to identify a statistically significant increase in such pulses during night side disk viewing, but found no meaningful indication of lightning. Gurnett et al. (1991) also examined the statistical distribution of pulse height measured using an HF receiver during the Venus flyby of the Galileo spacecraft and reported nine possible lightning pulses with pulse heights greater 
than 4 sigma of background. On the other hand, Gurnett et al. (2001) during two Venus flybys of the Cassini spacecraft found no evidence for sferics induced by lightning discharge (whereas the same instrumentation easily detected lightning emissions during a later Earth flyby). Hansell et al. (1995) detected several candidate lightning flashes using a 1.5-m ground-based telescope. The imaging rate of the charge-coupled device $(\mathrm{CCD})$ camera was 18.8 frames/s, which is not sufficiently rapid to take more than one frame for one lightning flash. Therefore, there remains some ambiguity in deciding whether the recorded light spot originated as lightning as cosmic rays or other unknown electrical noise could cause similar apparent features.

Krasnopolsky (1983a, b) observed random flashing during a single 70 -s period of observations of the Venera 9 visible airglow spectrometer; the corresponding Venera 10 instrument recorded no such flashes. If the Venera 9 signals indeed corresponded to lightning, they occurred in a region of $5 \times 10^{4} \mathrm{~km}^{2}$ with a flashing rate of $0.002 \mathrm{~km}^{-2} \mathrm{~s}^{-1}$, a mean flash duration of $0.25 \mathrm{~s}$, and a flash energy of $2 \times 10^{7} \mathrm{~J}$ in the visible range. Krasnopolsky (2006) reported detection of nitric oxide (NO) in Venus clouds and argued that lightning was the only plausible source of NO in the lower atmosphere (although he noted some uncertainty in relevant reaction rates and did not quantify meteoric production).

Among the best means to distinguish a lightning signature from possible noise is to inspect the light curve (or 'waveform') of a lightning flash sampled at a cadence sufficiently high compared to the expected duration of the flash. Such a waveform, whose shape is determined in part by the distribution of light propagation paths through the cloud, can be diagnostic of the depth at which the discharge occurs. On the other hand, a cosmic ray strike on the detector has an instantaneous rise and a decay constant was determined only by the instrumentation electronics.

\section{Instrumentation and observation strategy}

LAC is the first fast-sampling optical sensor designed to measure the light curve of lightning flashes on a planet other than the Earth. To achieve an adequate sampling rate, we sacrificed spatial resolution such that the avalanche photodiode (APD) format was only $4 \times 8$ pixels (totaling 32 pixels). Though the basic specifications of the instrument are the same as those listed in Takahashi et al. (2008), the sampling rate was modified from 50 to $31.25 \mathrm{kHz}$ for the flight model. Considering that the duration of optical lightning strokes measured using photometers of the Japanese Experiment Module (JEM)/Global Lightning and sprIte Measurements (GLIMS) instrument on the International Space Station is on the order of one millisecond, the LAC sampling interval of $30 \mu \mathrm{s}$ is considered adequate. The field of view is $8 \times 16^{\circ}$, corresponding to $700 \mathrm{~km} \times 1400 \mathrm{~km}$ on the surface of Venus at distance of $5000 \mathrm{~km}$, which is approximately the average of the closest range for all of the observations. The data recording and triggering logic were designed to accommodate various types of flash waveforms, given the unknown parameters of Venus lightning (Fig. 1). The triggering logic works using every pixel, and data were recorded only for the pixel triggered first. The recorded data length can be chosen from $2.048 \mathrm{~ms}$ to $2.096 \mathrm{~s}$. The time duration to define the background level ( $N$ in Fig. 1$)$ was from 0.02 to $8.192 \mathrm{~ms}$. The time gap between the last of the background sampling and the triggering timing $(t)$ was from 0.512 to $32.768 \mathrm{~ms}$. The threshold level was defined at 4 levels, while the sensitivity was also controlled by the high voltage to the APD. The necessary consecutive duration over the threshold for triggering $(n)$ was defined as $n / t$ from 1 to 8 . With this highly flexible combination of parameters, it is possible to trigger and store data for various types of optical phenomena, including not only typical cloud-to-ground or intra-cloud lightning discharges but also transient luminous events (TLEs) in the middle and upper atmosphere, termed sprites, elves, blue and gigantic jets, etc. The possibility of the occurrence of TLEs on Venus was discussed by Yair et al. (2009) and Pérez-Invernón et al. (2016). For the first trial, we chose a parameter set consisting of data length $=16.384 \mathrm{~ms}, N=0.512 \mathrm{~ms}, t=0.512 \mathrm{~ms}$, $n / t=2$, and a threshold $=2$ digits, which is optimized for normal lightning detection. Absolute calibration for the sensitivity of an individual pixel was conducted using a 2-m integrating sphere at the National Institute for Polar Research, Japan. The results showed a variation in relative sensitivity of approximately $\pm 50 \%$ from average. We use a typical value for the discussion here.

We selected the oxygen OI-777-nm line for lightning detection, which was expected to be the most prominent emission in a $\mathrm{CO}_{2}$-dominant atmosphere based on

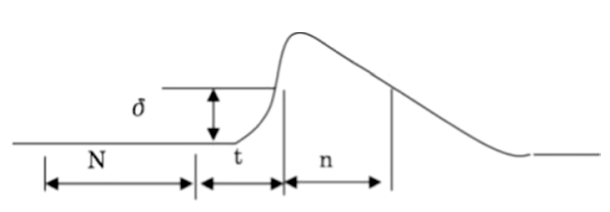

Fig. 1 Schematic drawing of triggering logic
Data length [ms]: 2.048, 16.384, 131.072, 2096

$\mathrm{N}[\mathrm{ms}]: 0.032,0.512,2.048,8.192$

$\mathrm{t}[\mathrm{ms}]: 0.512,2.048,4.096,8.192,32.768,262.144$

$\mathrm{n} / \mathrm{t}: 1,2,4,8$

d (threshold): 2 digit, 4 digit, 8 digit, 32 digit 
laboratory experiments (Borucki et al. 1985). The bandwidth (FWHM) and center wavelength of the LAC OI$777-\mathrm{nm}$ filter were 9.0 and $780.6 \mathrm{~nm}$, respectively. If the spacecraft is $5500 \mathrm{~km}$ above Venus surface, the threshold of triggering is approximately $1 / 20$ of the average luminosity of an Earth lightning flash and the instantaneous field of view (FOV) is $1 / 500$ of the whole globe. Here, we assume that approximately $40 \%$ of the total optical emission is from the OI-777-nm line according to the photographic spectra in Borucki et al. (1985). However, Krasnopolsky (2006) noted that OI $777 \mathrm{~nm}$ is approximately $3 \%$ of the total light in the more sensitive photoelectric spectra of Borucki et al. (1996), where the bright continuum dominates: In this case, our detection threshold would be higher, comparable to the average lightning on Earth.

\section{Orbital condition for observation and setup of high voltage for the APD}

Akatsuki was successfully inserted into orbit surrounding Venus during December 2016, but with a period of $\sim 10$ days, approximately $\sim 8$ times longer than the originally intended orbit. LAC can be powered only inside the umbra of Venus because the strong sunlight can cause damage to its detector. Therefore, the frequency of observation opportunities was reduced to $1 / 8$ of that of the original plan. Adding to this decrease in observational frequency, the elongated orbit resulted in a more rapid motion of spacecraft near periapsis where LAC can observe the night side disk at a close distance, meaning that the duration of observation for one revolution was shorter than that of the original. Indeed, most of the observational periods were approximately 20-30 min, while the original plan anticipated sessions a few times longer. Thus, the total observational period per year was reduced to less than $10 \%$ of that of the original plan because of the change in the orbital parameters. On the other hand, the successful insertion into orbit around Venus, 5 years later than the original plan, allowed for observations that would otherwise be completely impossible and promises with time to establish significant constraints on lightning activity on Venus.

The most important work in the start-up operation of LAC was the increase in high-voltage power supply to the APD. Because the high-voltage power supply unit had not been turned on since the launch in 2010, careful operation was required and the high voltage was increased only gradually, over 10 steps. As previously mentioned, the operation was conducted only during the short duration of the umbra of Venus; thus, each increment was performed during a different 10-day orbit. Figure 2 shows the relationship between the reverse voltage applied to the APD and the dark current of output for the operations from May 7 to November 9, 2016. The dark current was

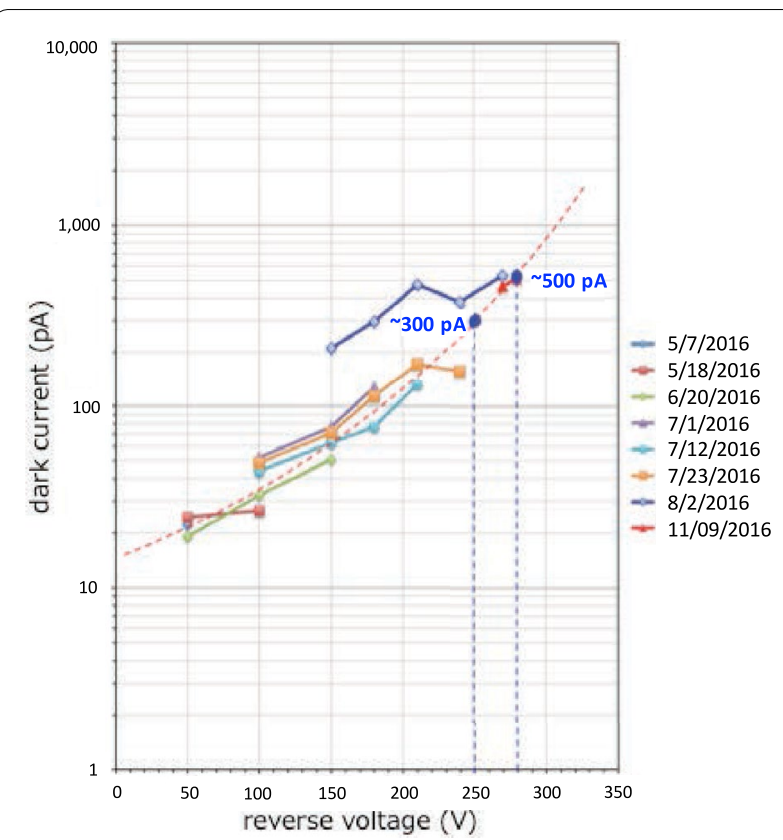

Fig. 2 Relationship between the reverse voltage applied to APD and the dark currents measured during 8 days from May 7 to November 9, 2016

approximately on the expected curve (red dotted line), while the deviation from the line was considered to be caused mainly by temperature fluctuations of the APD.

We chose $300 \mathrm{~V}$ as the applied voltage to the APD for regular lightning observations for the time being. Considering the level of dark current, the APD seems to be working properly without serious problems. At this voltage, the sensitivity of LAC is expected to be sufficient to detect an optical lightning flash with an intensity of 0.05 to 0.5 of the average Earth flash at a distance of $5500 \mathrm{~km}$, depending on its spectral distribution. At the longer range of our observations of $12,000 \mathrm{~km}$, the threshold could be as high as $2 \times$ that of the average Earth flash.

\section{Preliminary observations}

The lightning search at a nominal high voltage of $300 \mathrm{~V}$ was attempted 8 times in the lightning observation mode of LAC with each duration of $\sim 10$ to 30 min on December 1 , 2016. The distance to the Venus surface was in the range of $\sim 7500$ to $12,300 \mathrm{~km}$, meaning the averaged instant coverage was approximately $1 / 500$ of the whole globe and the threshold of the triggering changed by $\sim 1.6$ times in this case. There were some triggered events every pass. However, all showed a steep increase within two sampling intervals $(<63$ us, meaning they were caused by cosmic ray impact. Thus far, no signature of lightning has been identified. It was confirmed that all 32 pixels for lightning measurement detected 
cosmic ray events and the triggering logic for all pixels works properly. In addition, if some pixels were exposed to the limb of Venus illuminated by scattered sunlight, they recorded a reasonable light curve. Based on this and the increase in dark currents as the high voltage was ramped up, we consider LAC works overall as expected.

Figure 3 shows an example of the quick look data from one operation. The upper panel indicates the distance

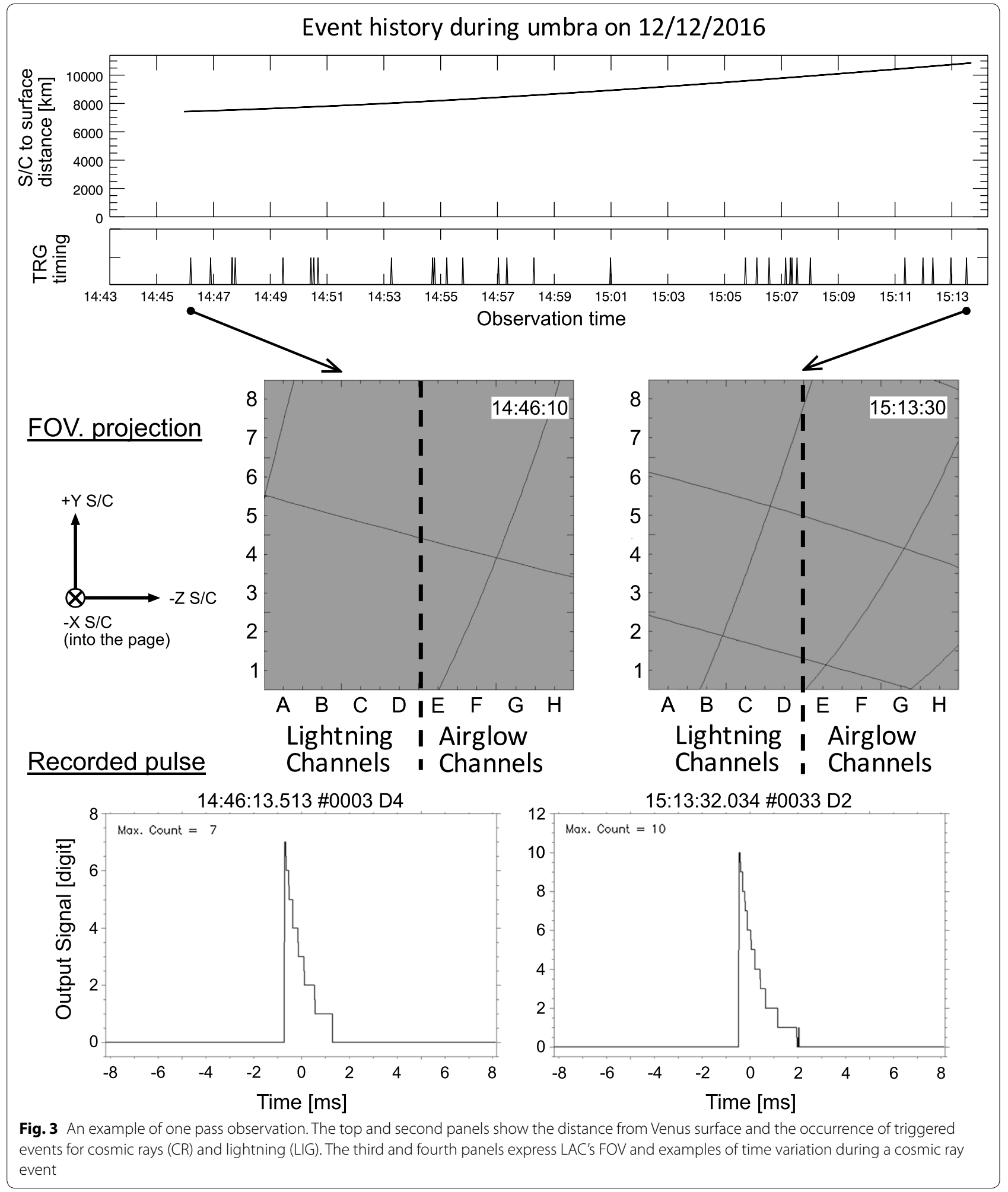


from the Venus surface and the second the time series of triggered pulses. At each pulse time, we checked the projection of LAC's FOV onto Venus. (The lightning observing area is rows A-D.) The bottom plots are examples of waveforms caused by cosmic rays.

The heights of the cosmic ray pulses are consistent with the triggering level set in the lightning observation mode, implying a normal condition of triggering logic and hardware. All the curves show the same decay period of $1.8 \mathrm{~ms}$, which was expected from the design of the electronic circuit and temperature of the APD. Considering these conditions, the health and performance of LAC are quite sufficient to capture a lightning flash if it were to occur inside the FOV at brightness greater than the threshold of the instrument as designed.

\section{Future work}

Given that cosmic ray events are triggered at our chosen threshold values and recorded properly, and the dim light near the limb caused triggering by the designed logic and provided saturated signals, the optical and electronic performance of LAC-including its sensitivity-appears to be normal, even after prolonged flight in space. However, it would be desirable to measure the absolute sensitivity from on-orbit observational data on Venus in the future. Among the ideas is to measure the Earth which is the only celestial target sufficiently bright to detect in the lightning observation mode of LAC. Another possibility would be to observe the limb together with the ultraviolet imager (UVI) onboard Akatsuki and compare it to the UVI image whose sensitivity can be calculated from star images.

We expect to continue the lightning search operations for several years. To increase the possibility of capturing events, we plan to concentrate the dusk area of the night side disk where the thunderstorm activity may be higher than at other local times according to experience on Earth. Indeed, 5 of the 8 possible lightning events reported by Hansell et al. (1995) and Krasnopolsky (1983a, b) were found in the dusk region on Venus. We also plan to focus our search for optical transients near candidate volcanos: Volcanic plumes might result in convective activity in clouds, or (as on Earth) there may be discharge within the plume itself. Hashimoto and Imamura (2001) discuss the possibility of volcanic detection using an infrared camera onboard Akatsuki. Russell (1991) reviewed possible lightning sources including active volcanos.

\section{Conclusion}

Initial operations of LAC including the operational level of high voltage applied to the APD have been successfully completed. The threshold of optical detection is $0.05-2$ times that of the averaged intensity of Earth's lightning. The performance of LAC seems nominal, as evidenced by the detection and correct recording of cosmic rays and dim luminosity near the limb. Though the duty cycle of the lightning observation time in the present orbit is $>10$ times less than that in the originally planned orbit of Akatsuki, continued operation over the coming years will provide a statistically robust dataset.

\section{Authors' contributions}

YT contributed to the entire design of the research and equipment related to the LAC instrument onboard Akatsuki. MS and MI conducted the operation of LAC and data analysis. RL participated in the essential scientific discussion and helped write the manuscript as a native speaker. YY, KA, and GF contributed to the strategy of observation and scientific discussion. MN worked as a project manager on the Akatsuki project, supervising scientific orientation. NI was in charge of satellite development and operations based on the understanding of the scientific purposes, providing advice to the LAC operation. TA, TI, MS, AY, TS, MY, HA: contributed to project management, understanding the scientific target of LAC. TS worked as project scientist on the Akatsuki project, supervising scientific analysis. $\mathrm{CH}$ was in charge of orbital design and operation based on an understanding of the scientific purposes. GH, NH, SM, YY, KO, SO: conducted satellite data processing. TF, KS, HK: contributed to satellite payload operation. All authors read and approved the final manuscript.

\begin{abstract}
Author details
${ }^{1}$ Graduate School of Science, Hokkaido University, Kita 10 Nishi 8, Kita-ku, Sapporo, Hokkaido 060-0810, Japan. ${ }^{2}$ Johns Hopkins University Applied Physics Lab, 11100 Johns Hopkins Road, Laurel, MD 20723, USA. ${ }^{3}$ School of Sustainability, Interdisciplinary Center Herzliya (IDC), P. O. Box 167, 46150 Herzliya, Israel. ${ }^{4}$ Physics Teaching Laboratories, Oxford University, Denys Wilkinson Building, Keble Rd, Oxford OX1 3RH, UK. ${ }^{5}$ Space Research Institute, Austrian Academy of Sciences, Schmiedlstr. 6, 8042 Graz, Austria. ${ }^{6}$ Institute of Space and Astronautical Science, Japan Aerospace Exploration Agency, 3-1-1 Yoshinodai, Chuo-ku, Sagamihara, Kanagawa 252-5210, Japan. ${ }^{7}$ Department of Space and Astronautical Science, School of Physical Sciences, SOKENDAI, 3-1-1 Yoshinodai, Chuo-ku, Sagamihara, Kanagawa 252-5210, Japan. ${ }^{8}$ Graduate School of Frontier Sciences, The University of Tokyo, Kiban-tou 4H7, 5-1-5 Kashiwanoha, Kashiwa, Chiba 277-8561, Japan. ${ }^{9}$ Department of Earth Science, Okayama University, 3-1-1 Tsushimanaka, Kita, Okayama 700-8530, Japan.

${ }^{10}$ School of Computer Science and Engineering, The University of Aizu, 90 Kami-lawase, Tsuruga, Ikki-machi, Aizu-Wakamatsu, Fukushima 965-8580, Japan. ${ }^{11}$ Department of Earth and Planetary Science, Graduate School of Science, The University of Tokyo, Hongo 7-3-1, Bunkyo-ku, Tokyo 113-0033, Japan. 12 Planetary Exploration Research Center, Chiba Institute of Technology, 2-17-1 Tsudanuma, Narashino, Chiba 275-0016, Japan. ${ }^{13}$ Department of Physics, Rikkyo University, 3-34-1 Nishi-lkebukuro, Toshima-ku, Tokyo 171-8501, Japan. ${ }^{14}$ School of Engineering, University of Shiga Prefecture, 2500 Hassaka-cho, Hikone, Shiga 522-8533, Japan. ${ }^{15}$ Faculty of Science, Kyoto Sangyo University, Motoyama, Kamigamo, Kita-ku, Kyoto, Kyoto 603-8555, Japan. ${ }^{16}$ Department of Information Engineering, National Institute of Technology, Matsue College, 14-4 Nishi-Ikuma, Matsue, Shimane 690-8518, Japan. ${ }^{17}$ Japan Agency for Marine-Earth Science and Technology, 3173-25 Showa-machi, Kanazawa-ku, Yokohama, Kanagawa 236-0001, Japan. ${ }^{18}$ School of Commerce, Senshu University, 2-1-1 Higashimita, Tama-ku, Kawasaki, Kanagawa 214-8580, Japan.
\end{abstract}

\section{Acknowledgements}

Akatsuki project activity is supported by the Japan Space Exploration Agency (JAXA). The authors would like to express their thanks to all of the Akatsuki project supporting members in JAXA and universities. In addition, the creative contributions of Meisei Electric Co., Ltd., Nikon Co., and Hamamatsu Photonics K.K. in designing and fabricating the instrumentation are greatly appreciated. $\mathrm{RL}$ acknowledges the support of the National Aeronautics and Space Administration Voluntary Consensus Organization Participating Scientist Program, Grant NNX16AC78G. 


\section{Competing interests}

The authors declare that they have no competing interests.

Ethics approval and consent to participate

The authors declare that there is no ethical conflict in this study.

\section{Publisher's Note}

Springer Nature remains neutral with regard to jurisdictional claims in published maps and institutional affiliations.

Received: 1 July 2017 Accepted: 14 April 2018

Published online: 25 May 2018

\section{References}

Borucki WJ, Dyer JW, Thomas GZ, Jordan JC, Comstock DA (1981) Optical search for lightning on Venus. Geophys Res Lett 8:233-236. https://doi. org/10.1029/GL008i003p00233

Borucki WJ, Mc Kenze RL, McKay CP, Duong ND, Boac DS (1985) Spectra of simulated lightning on Venus, Jupiter, and Titan. Icarus 64:221-232. https ://doi.org/10.1016/0019-1035(85)90087-9Medline

Borucki WJ, Dyer JW, Phillips JR, Phan P (1991) Pioneer Venus orbiter search for Venusian lightning. J Geophys Res 96:11033-11043. https://doi. org/10.1029/91JA01097

Borucki WJ, McKay CP, Jebens D, Lakkaraju HS, Vanajakshi CT (1996) Spectral irradiance measurements of simulated lightning in planetary atmospheres. Icarus 123(2):336-344

Gurnett DA, Kurith WS, Roux A, Gendrin R, Kennel CF, Bolton SJ (1991) Lightning and plasma wave observations from the Galileo flyby of Venus, Science 253:1522-1525. https://doi.org/10.1126/science.253.5027.1522
Gurnett DA, Zarka P, Manning R, Kurth WS, Hospodarsky GB, Averkamp TF et al (2001) Non-detection at Venus of high-frequency radio signals characteristic of terrestrial lightning. Nature 409:313-315. https://doi. org/10.1038/35053009

Hansell SA, Wells WK, Hunten DM (1995) Optical detection of lightning on Venus. Icarus 117:345-351

Hashimoto G, Imamura T (2001) Elucidating the rate of volcanism on Venus: detection of lava eruptions using near-infrared observations. Icarus. https ://doi.org/10.1006/icar.2001.6713

Krasnopolsky VA (1983a) Venus spectroscopy in the 3000-8000. A region by Veneras 9 and 10. In: Hunten DM et al (eds) Venus. Univ. of Arizona Press, Tucson, pp 459-483

Krasnopolsky VA (1983b) Lightnings and nitric oxide on Venus. Planet Space Sci 31:1363-1369

Krasnopolsky VA (2006) A sensitive search for nitric oxide in the lower atmospheres of Venus and Mars: detection on Venus and upper limit for Mars. Icarus 182:80-91

Pérez-Invernón FJ, Luque A, Gordillo-Vázquez FJ (2016) Mesospheric optical signatures of possible lightning on Venus. J Geophys Res Space Phys. https://doi.org/10.1002/2016JA022886

Russell CT (1991) Venus lightning. Space Sci Rev 55:317-356

Takahashi T, Yoshida J, Yair Y, Imamura T, Nakamura M (2008) Lightning detection by LAC onboard the Japanese Venus climate orbiter, planet-C. Space Sci Rev. https://doi.org/10.1007/s11214-008-9400-x

Yair Y, Takahashi Y, Yaniv R, Ebert U, Goto Y (2009) A study of the possibility of sprites in the atmospheres of other planets. J Geophys Res. https://doi. org/10.1029/2008JE003311

\section{Submit your manuscript to a SpringerOpen ${ }^{\circ}$ journal and benefit from:}

- Convenient online submission

- Rigorous peer review

- Open access: articles freely available online

- High visibility within the field

- Retaining the copyright to your article

Submit your next manuscript at $\boldsymbol{\nabla}$ springeropen.com 